\title{
ON LINEAR BIREFRINGENCE ANOMALIES IN CRYSTALS OF TRIGLYCINE FAMILY
}

\section{J. Przeslawsiki}

Institute of Experimental Physics, University of Wrocław

Pl. Maxa Borna 9, 50-204 Wrocław, Poland

(Received December 10, 1998; in final form April 30, 1999)

Temperature dependence of the linear birefringence in TGS crystal family has been studied with a high temperature resolution. Temperature derivatives of the linear birefringence are compared with the specific heat transients measured for triglycine sulphate, triglycine selenate, and triglycine fluoberyllate crystals. Detailed studies revealed an anomaly of the linear birefringence at about $310 \mathrm{~K}$ for two cuts ( $a^{*}$ - and $c$-cut) of the TGS crystal.

PACS numbers: $77.80 . \mathrm{Bh}, 78.20 . \mathrm{Fm}$

\section{Introduction}

Linear birefringence (l.b.) change is a property that can be measured very precisely and can serve as an especially valuable tool in phase transitions studies $[1,2]$. The l.b. changes in triglycine sulphate (TGS), triglycine fluoberyllate (TGFB), and triglycine selenate (TGSe) crystals were measured by several investigators [3-8]. All the published data were collected with a rather poor temperature resolution. The aim of our paper is to present the I.b. measurements performed with a precise modulation technique and very low temperature gradient at the phase transition region.

\section{Experimental}

Since late fifties the triglycine family of ferroelectric crystals has been, as perovskites, probably the most intensely studied. Two (TGS and TGFB) of three crystals used in below reported experiments are regarded as model second-order phase transition materials. Ferroelectric transition in the TGSe is described as very close to the tricritical one [9]. A crystal structure in paraelectric and ferroelectric phases is monoclinic, the space group changes from $P 2_{1} / m$ to $P 2_{1}$, respectively. The spontaneous polarization is directed along the $b$-axis. Samples of chosen cuts (ferroelectric $b_{-}, c_{-}$, and $a^{*}$-cut) were prepared from TGS, TGFB, and TGSe crystals. The choice was connected with location of the optical indicatrix in all the crystals mentioned. The orientation of the indicatrix axes was checked by a polarization microscope. The optical plane forms a $93^{\circ}$-angle with the $c$-axis and approximately coincides with the plane (102) (according to the Wood and Holden system of crystallographic axes [10]). Linear birefringence changes were measured 
by the modified rotating-analyzer method [11]. A modulated signal was registered with a lock-in nanovoltmeter. A He-Ne laser $(\lambda=632.8 \mathrm{~nm})$ served as a light source. Fluctuations of the laser beam intensity were also registered and the output signal was divided by the input intensity. The apparatus was fully controlled by the PC. Data were collected during heating and cooling processes with a temperature rate of $0.1 \mathrm{~K} / \mathrm{min}$ at the phase transition region. Before the cooling stage, the samples were rejuvenated at a temperature $T_{\mathrm{C}}+25 \mathrm{~K}$ for several hours with shorted electrodes deposited on the $b$ planes $\left(T_{\mathrm{C}}-\right.$ the Curie temperature; for the TGSe: $T_{\mathrm{C}} \approx 295 \mathrm{~K}$, for the TGS: $T_{\mathrm{C}} \approx 322 \mathrm{~K}$, and for the TGFB: $T_{\mathrm{C}}=346 \mathrm{~K}$ ). Since the rotating-analyzer measures changes in the retardation $\Delta n d$ (where $\Delta n-$ the linear birefringence, $d-$ the sample thickness), the Ehringhaus compensator should be used to get an absolute scale in the birefringence.

\section{Results and discussion}

Although the three chosen crystals are isomorphic, there is a significant difference in a temperature dependence of the linear birefringence changes $\Delta\left(\Delta n_{b}\right)$ measured for the ferroelectric samples (b-cuts) (Fig. 1). A very peculiar behaviour has been found for the TGFB crystal (Fig. 3). The temperature transients of the $\Delta\left(\Delta n_{b}\right)$ of the TGS and TGSe are similar but the features specific to the ferroelectric transitions in both materials can be seen more distinctly when the linear birefringence increments $\delta\left(\Delta n_{b}\right)$ are compared. These increments are obtained by subtraction from the $\Delta\left(\Delta n_{b}\right)(T)$-dependence, the paraelectric, linear or slightly nonlinear part of the $\Delta\left(\Delta n_{b}\right)(T)$, prolonged to the ferroelectric phase. Spontaneous l.b. increments induced in the transition are proportional to the square of the order parameter (the spontaneous polarization) for the proper ferroelectric transitions $\delta\left(\Delta n_{b}\right) \sim\left(T_{\mathrm{C}}-T\right)^{2 \beta}$. The order parameter critical index $\beta$ could be then estimated from the slope of the plot $\log \delta\left(\Delta n_{b}\right)$ versus $\log \left(T_{\mathrm{C}}-T\right)$ (Fig. 2).

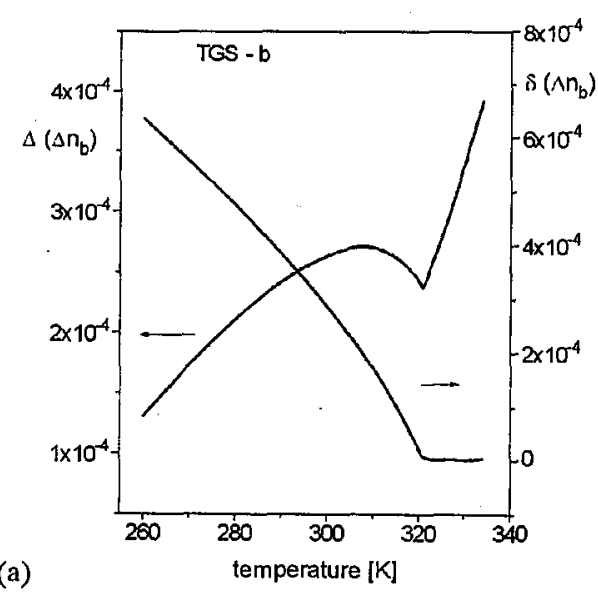

Fig. 1. Temperature dependence of the linear birefringence changes $\Delta\left(\Delta n_{b}\right)$ and increments $\delta\left(\Delta n_{b}\right)$ in TGS (a) and TGSe (b) crystals.

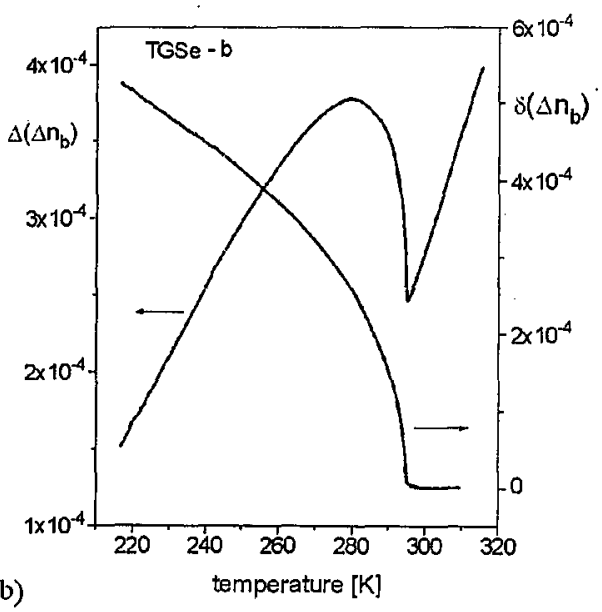

(b) 

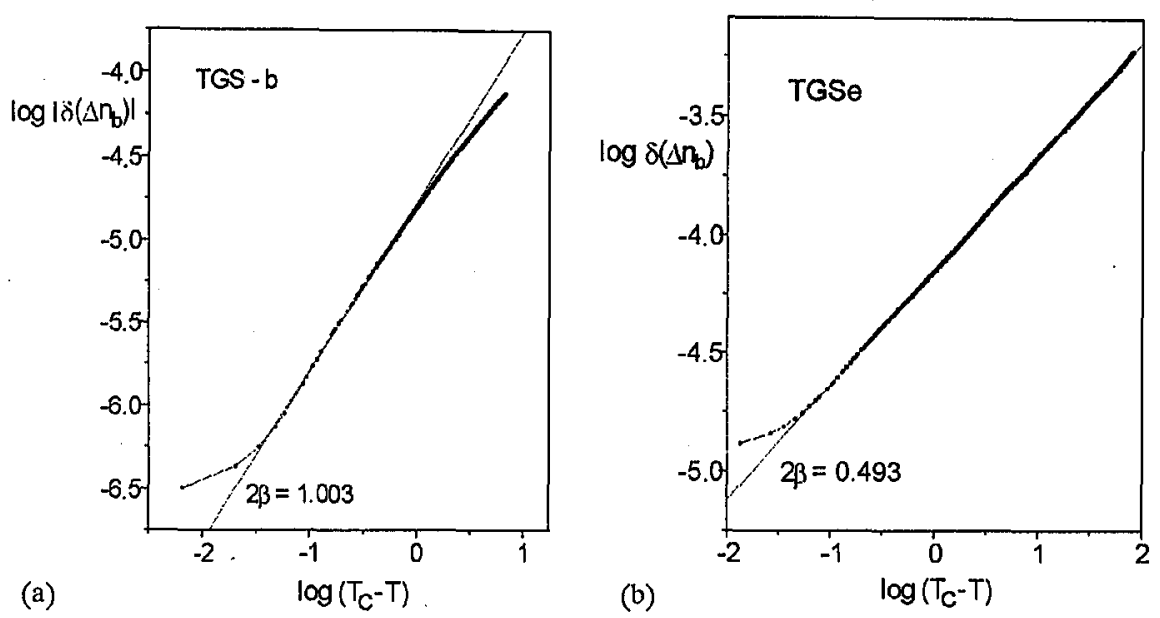

Fig. 2. Log-log plots of $\delta\left(\Delta n_{b}\right)$ versus $\left(T_{C}-T\right)$ for TGS (a) and TGSe (b). $\beta$ is the order parameter critical index.

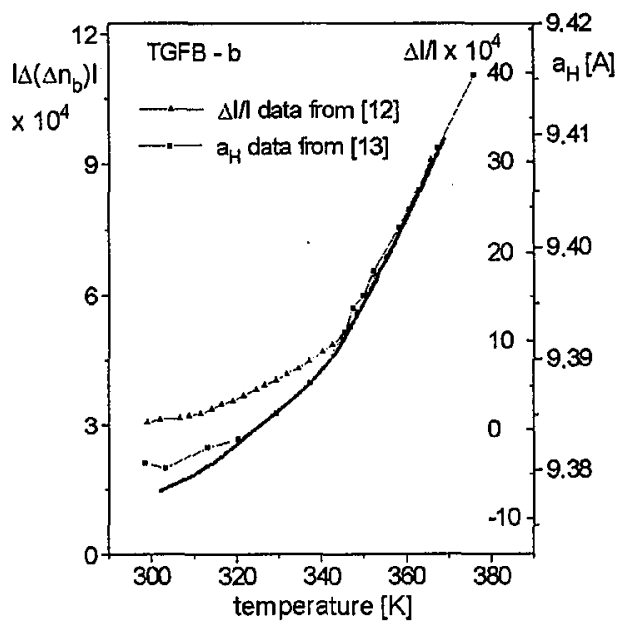

Fig. 3. Comparison of the linear birefringence change $\Delta\left(\Delta n_{b}\right)(T)$ and the dilation $\left(\Delta l_{a \mathrm{H}} / l\right)(T)$ for the TGFB crystal.

The index value found for the TGS crystal was very close to the classical one: 0.502 , whereas for the TGSe was 0.247 , which confirmed the tricritical character of the ferroelectric transition in the TGSe. The $\Delta\left(\Delta n_{b}\right)(T)$-dependence obtained for the TGFB differs strongly from the respective transients measured for other crystals of glycine family. It was rather straightforward to compare the l.b. changes with dilation data $[12,13]$. The dilation in the TGFB along the $a_{\mathrm{H}}$-axis $\left(a_{\mathrm{H}}-\right.$ axis of the HOP system [14]) differs also from the same dilation measured for the TGS and TGSe crystals. We tried to check how close these two transients, i.e. 
$\Delta\left(\Delta n_{b}\right)(T)$ and $\left(\Delta l_{a \mathrm{H}} / l\right)(T)$, are proportional to each other for the TGFB crystal, Fig. 3 ; it seems clear that a quality and scattering of dilation data are of decisive importance. Data of Warkusz [13] were obtained from lattice constants measurements by the Bond method, whereas an interference dilatometry was used in the experiment of Stankowski and Malinowski [12]. It is known [15] that a maximal polarizability is directed approximately along the $a^{*}$-direction in the TGS crystal. A slightly deformed $\mathrm{SO}_{4}^{2-}$-ion is bonded by the strongest (shortest) hydrogen bond with a glycine I. The very similar situation can be found in the TGSe. In the TGFB crystal the whole ion is exchanged; the $\mathrm{BeF}_{4}^{2-}$-ion is a little larger than the sulphate ion. The hydrogen bonds between the glycine $\mathrm{I}$ and $\mathrm{BeF}_{4}^{2-}$-ions are shorter (stronger) than the respective bonds in the TGS structure. The essential role of the carbonyl chromophores of glycine $\mathrm{I}$ and deformation of $\mathrm{SO}_{4}^{2-}$-ion in origin of the optical activity in the TGS in the ferroelectric phase was also established [16]. The linear birefringence increment $\delta\left(\Delta n_{b}\right)$ in the TGFB is quasi linear in the ferroelectric phase but the transition region is smeared, diffused. The respective behaviour for the $a^{*}$-cut is presented in Fig. 4. The transition temperature and the critical index $(\beta \approx 0.501)$ can be clearly determined.

It has been known for ferromagnets [17-19] that the temperature derivative of the linear birefringence can be scaled with the $C_{\mathrm{m}}$ (the magnetic specific heat) at the transition region. It was shown that the birefringence method is considerably less sensitive to phonon contribution than specific heat techniques. Similar attempts to estimate specific heat behaviour from the linear birefringence changes were performed also for ferroelastic transitions [20, 21]. Our purpose was to check this dependence for the ferroelectric crystals. We presented an analogous behaviour of the temperature derivative of the linear birefringence

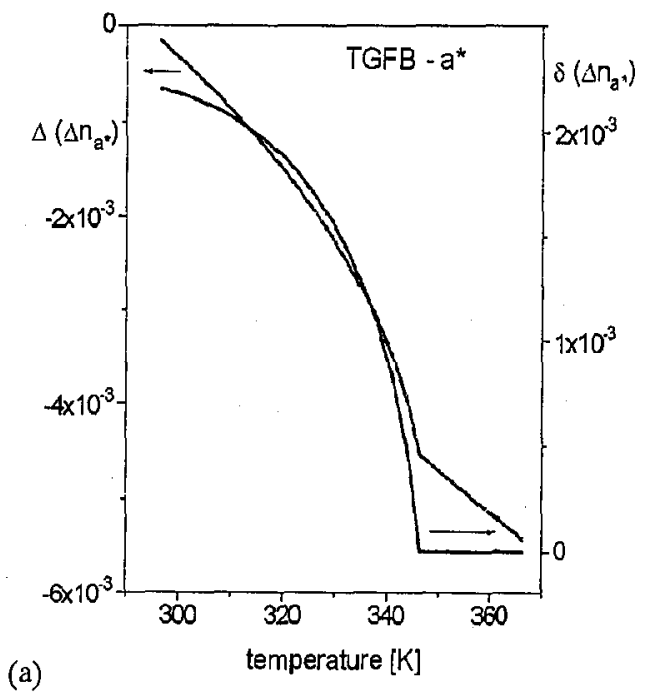

(a)

Fig. 4. Linear birefringence change $\Delta\left(\Delta n_{a^{*}}\right)(T)$, the increment $\delta\left(\Delta n_{a^{*}}\right)$ (a) and the $\log -\log$ plot of $\delta\left(\Delta n_{a^{*}}\right)$ versus $\left(T_{\mathrm{C}}-T\right)$ (b) for TGFB crystal. 
and $\Delta C_{p}$ in the $\left(\mathrm{CH}_{3} \mathrm{NH}_{3}\right)_{5} \mathrm{Bi}_{2} \mathrm{Cl}_{11}$ (MAPCB) ferroelectric crystal [22]. Assuming in the simplest approximation: $\Delta S^{E}=(A / 2) P_{s}^{2}(T)$ (where $S^{E}$ - the entropy $(E=0), A(T)=A\left(T-T_{\mathrm{C}}\right)-$ the temperature dependent coefficient in the free energy Landau expansion), $\Delta C^{E} / T=(A / 2) \mathrm{d} P_{s}^{2} / \mathrm{d} T\left(\Delta C^{E}\right.$ - the increment of the specific heat). This dependence of $\Delta S$ on the square of the order parameter can be quite useful if the order parameter is not a measurable quantity. It provides for proportionality of $\Delta C / T$ with temperature derivatives of all quantities quadratically coupled with the order parameter even when they do not belong to the compliance matrix, like the spontaneous birefringence [23]. Since the linear birefringence increment $\delta(\Delta n) \propto P_{s}^{2}$, then the temperature derivative of linear birefringence $\mathrm{d}(\Delta n) / \mathrm{d} T \propto \Delta C / T$. In the following figures (Fig. 5) a comparison of the temperature derivatives of the linear birefringence $\mathrm{d}(\Delta n) / \mathrm{d} T$ and the specific heat increments $\Delta C / T$ is presented for our three crystals. Specific heat data
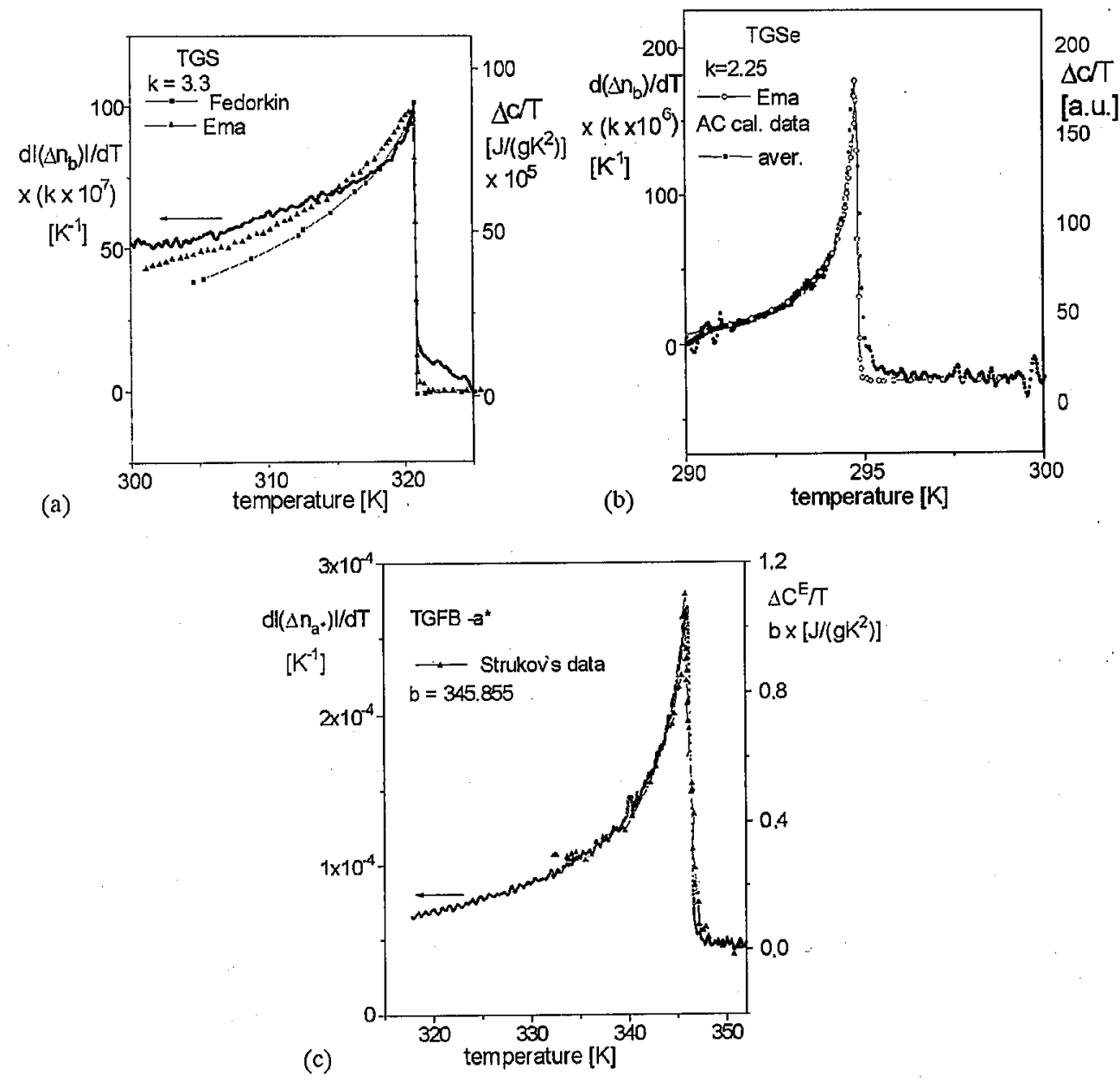

Fig. 5. Comparison of temperature derivatives of linear birefringence $\mathrm{d}(\Delta n) / \mathrm{d} T$ and the specific heat increment $\Delta C / T$ for TGS (a), TGSe (b), and TGFB (c) crystals. 
were taken from the best reports published by Ema et al. [24], Fedorkin et al. [25]. for the TGS crystal, by Ema et al. [26] for the TGSe crystal, and by Strukov et al. [27] for the TGFB. It should be pointed out that the specific heat data given by various authors differ significantly, e.g. the data for the TGS crystal [28]. We took into account the temperature derivatives of $\Delta n_{b}$ for the TGS and TGSe crystals and $\Delta n_{a^{*}}$ for the TGFB. The agreement is quite good for the TGFB, still acceptable for the TGSe, and rather bad for the TGS crystal. There would be probably much better agreement if the data for the linear birefringence and the specific heat measurements were collected for the same sample.
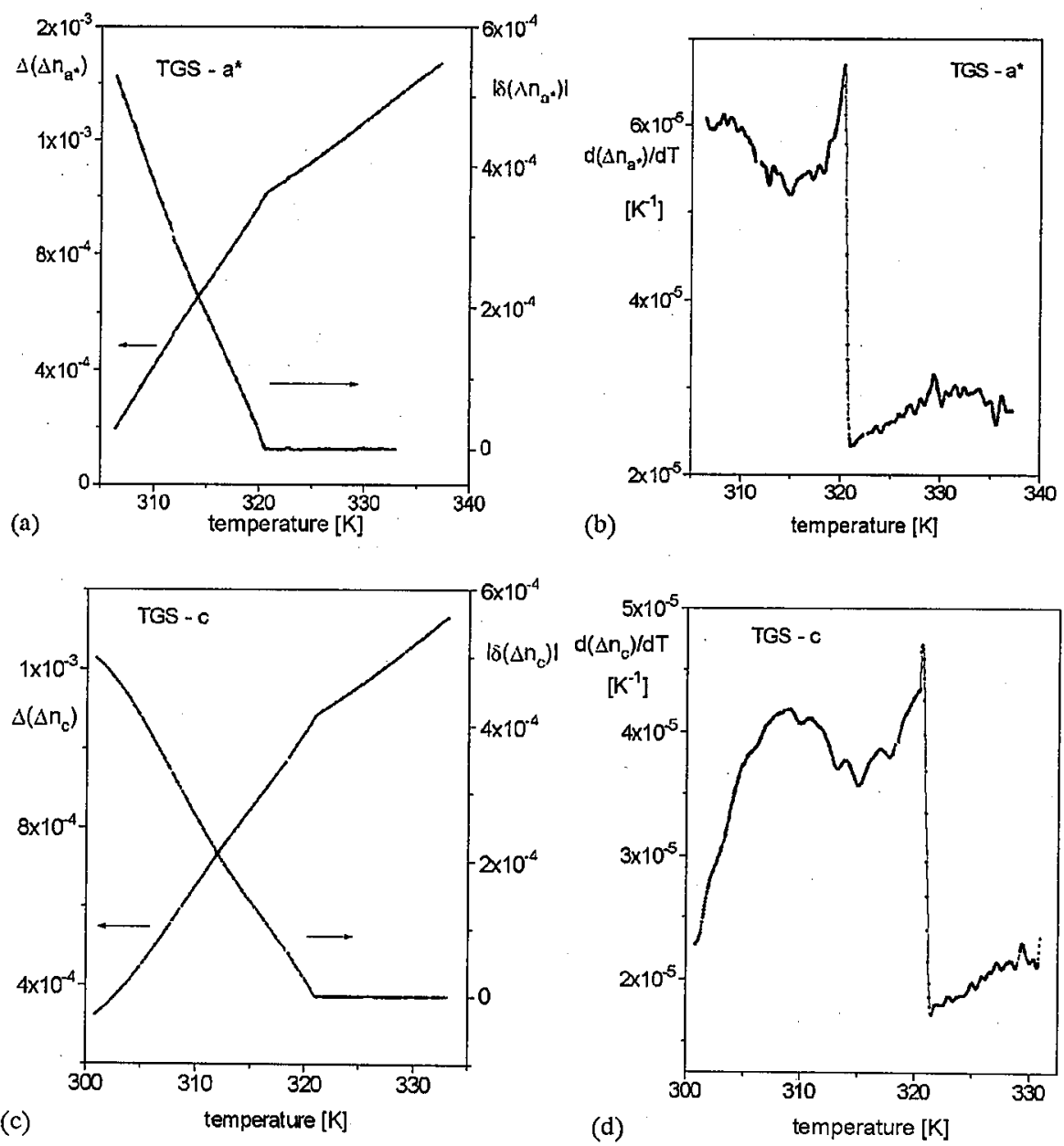

Fig. 6. Linear birefringence change, the l.b. increment (a), and its temperature derivative (b) for the $a^{*}$-cut; the l.b. change, the l.b. increment (c), and its temperature derivative (d) for the c-cut of TGS crystal. 
We have found also an anomalous temperature dependence of the linear birefringence and as a consequence - of the temperature derivative of the l.b. in the TGS crystal for the c-cut (the cut perpendicular to the optic plane). As it is shown in Fig. 6, temperature derivatives of the linear birefringence for the $c$-, and $a^{*}$-cut exhibit a cusp-shape maximum at a temperature of about $309-310 \mathrm{~K}$. In order to find an origin of the cusp maximum an external field was applied to the samples along the $b$-direction. Even while changing sign and intensity of the field we have observed only a diffusion of the phase transition, as it is the case when a bias field in the crystal is significant, but not a shift in position and height of the cusp maximum on the linear birefringence derivative curve. There were many examples of anomalies observed at about 308-310 K in the TGS crystal. It. was shown that the domain structure density exhibits a local maxinium at about $312 \mathrm{~K}$ [29]. Domain structure rebuilding was assumed as a reason for a relaxation time anomaly [30], and a jump in conductivity and in exoelectron emission was also reported at the same temperature range [31]. SEM studies of the TGS surface revealed a disappearing of contrast at about $310 \mathrm{~K}$ [32]. Friction force microscopy investigations on the cleaved TGS samples [33] confirmed the disappearing of the domain contrast at the same temperature. The linear birefringence in TGS should not depend on domain polarization, even if we tried to keep the sample as the monodomain, still the cusp-like transient of the linear birefringence derivative has been observed.

\section{Conclusions}

Three isomorphic crystals: TGS, TGSe, and TGFB, revealed different temperature behaviour of l.b. changes. Possible origins of the l.b. anomalous transient for TGFB were presented.

Order parameter critical indices were estimated and the tricritical character of the ferroelectric transition in the TGSe crystal was evidenced. Satisfactory agreement between the specific heat and the temperature derivative of the l.b. was checked for the TGFB and TGSe crystals.

Precise l.b. measurements enabled the detection of the slight but clearly visible (on the l.b. derivative transient especially) anomaly at about $310 \mathrm{~K}$ for the TGS crystal.

It can be concluded that linear birefringence studies performed for seemed "well-known" model ferroelectric materials have revealed anomalous features that still need more detailed investigations.

\section{Acknowledgments}

Author is thankful to the University of Wrocław for support under the grant 2016/IFD/98.

\section{References}

[1] J. Fousek, J. Petzelt, Phys. Status Solidi A 55, 11 (1979).

[2] J. Ferré, G.A. Gehring, Rep. Prog. Phys. 47, 513 (1984).

[3] N.R. Ivanov, S.Ya. Bendersky, I.T. Tukhtasunov, Izv. Akad. Nauk 39, 798 (1975). 
[4] O.G. Vlokh, L.F. Luciv-Schumsky, Ukr. Fiz. Zhurn. 11, 1009 (1966).

[5] W.J. Kusto, Ber. Bunsenges. Phys. Chem. 87, 284 (1983).

[6] M. Koralewski, A.M. Glazer, A. Czarnecka, Ferroelectrics 80, 261 (1988).

[7] J. Kobayashi; Y. Uesu, H. Takehara, J. Appl. Cryst. 16, 212 (1983).

[8] J. Etxebarria, J. Ortega, T. Breczewski, J. Phys., Condens. Matter 4, 6851 (1992).

[9] T. Iglesias, B. Noheda, G. Gallego, J.R. Fernandez del Castillo, G. Lifante, J. Gonzalo, Europhys. Lett. 28, 91 (1994).

[10] E. Wood, A.N. Holden, Acta Crystallogr. 10, 145 (1957).

[11] I.G. Wood, A.M. Glazer, J. Appl. Crystallogr. 13, 2127 (1980).

[12] J. Stankowski, W. Malinowski, Acta Phys. Pol. A 58, 773 (1980).

[13] F. Warkusz, Ph.D. Thesis, Inst. Low Temp. Str. Res. Pol. Acad. Sci., Wrocław 1971 , p. 35.

[14] S. Hoshino, Y. Okaya, R. Pepinsky, Phys. Rev. 115, 323 (1959).

[15] N.R. Ivanov, L.A. Shuvalov, D.D. Kislovsky, Dokl. Acad. Nauk SSSR 171, 1092 (1966).

[16] M. Koralewski, S. Habryło, Ferroelectrics 46, 13 (1982).

[17] I.R. Jahn, Phys. Status Solidi B 57, 681 (1973).

[18] D.P. Belanger, P. Nordblad, A.R. King, V. Jaccarino, L. Lundgren, O. Beckman, J. Magn. Magn. Mater. 31-34, 1095 (1983); D.P. Belanger, A.R. King, V. Jaccarino, J.L. Cardy, Phys. Rev. B 28, 2522 (1983).

[19] T. Levola, W. Kleemann, Phys. Rev, B 32, 4697 (1985).

[20] E. Salje, B. Wruck, Phys. Rev. B 28, 6510 (1983).

[21] M.V. Gorev, S.V. Melnikova, I.N. Florov, Fiz. Tverd. Tela 29, 2084 (1987).

[22] J. Przesławski, J. Phys., Condens. Matter 7, 4169 (1995).

[23] A. Fouskova, Ferroelektrizität 81/1, 10 (1981).

[24] K. Ema, K. Hamano; Y. Ikeda, J. Phys. Soc. Jpn. 46, 345 (1979); K. Ema, K. Hamano, Ferroelectrics 20, 193 (1978).

[25] W.A. Fedorkin, S.A. Taraskin, B.A. Strukov, E.A. Sorokina, Fiz. Tverd. Tela 22, 3544 (1980).

[26] K. Ema, K. Hamano, K. Kurihara, I. Hatta, J. Phys. Soc. Jpn. 43, 1954 (1977).

[27] B.A. Strukov, S.A. Taraskin, W. Kopcik, Zh. Eksp. Teor. Fiz. 51, 1037 (1966).

[28] S. Ramos, J. del Cerro, M. Zamora, Phys. Status Solidi A 61, 307 (1980).

[29] J. Stankowska, E. Czosnowska, Acta Phys. Pol. A 43, 641 (1973).

[30] B.N. Prasolov, I.A. Safonova, Ferroelectrics 157, 99 (1994).

[31] S.D. Milovidova, A.S. Sidorkin, A.M. Savinov, A.I. Maslakov, Fiz. Tverd. Tela 28, 2541 (1986); A.S. Sidorkin, Pv. Loginov, O.D. Gurin, A.M. Savinov, N.Yu. Ponomareva, S.D. Milovidova, J. Kor. Phys. Soc. 32, S793 (1998).

[32] D. Averty, R. Le Bihan, Ferroelectrics 140, 17 (1993).

[33] A. Correia, J. Massanell, N. Garcia, A.P. Levanyuk, A. Zlatkin, J. Przesławski, Appl. Phys. Lett. 68, 2796 (1996). 